\title{
Mobile remote-presence devices for point-of-care health care delivery
}

\author{
Ivar Mendez MD PhD, Michiel C. Van den Hof MD
}

Competing interests:

Ivar Mendez and Michiel

Van den Hof have

collaborated with InTouch

Health, as well as other

high-tech companies, and

worked with their devices in

a number of remote-

presence projects. However,

they are not paid

consultants or investors,

and have never received

financial remuneration for their projects. No products were provided by InTouch Health free of charge or at a reduced price for studies described in this paper.

This article has been peer reviewed.

Correspondence to: Ivar Mendez,mendez@dal.ca

CMAJ 2013. DOI:10.1503 /cmaj.120223
$\mathrm{T}$ imely access to effective medical care is a substantial challenge common to both developed and developing countries. In industrialized nations, barriers to medical care caused by distance and lack of adequate health care infrastructure and medical expertise have a negative impact on the provision of health care to vulnerable populations in underserviced remote communities. These barriers have an even greater impact in the developing world, where large segments of the population may lack access to primary health care services.

The rapid expansion in computer and telecommunications technology has enabled the development of solutions that may help address these challenges. Although major advances in the use of Internet-based telemedicine applications have occurred in the past decade, substantial challenges need to be overcome for telemedicine to achieve its full potential. ${ }^{1}$ Lack of Internet connectivity beyond large urban populations, unavailability of power supply, and limited local expertise in computers and technology are major obstacles in the implementation of effective telemedicine solutions, especially in resource-

\section{KEY POINTS}

- Remote-presence mobile technology can connect a primary care physician or specialist to the point-of-care setting, promoting real-time assessment and monitoring of the patient through wireless networks.

- Although pilot studies are underway in this emerging field, currently, there is limited literature supporting the wider use of remote-presence mobile technology.

- With continued technological advancement, our ability to use these devices to deliver health-behaviour interventions, perform point-ofcare diagnoses and provide treatment in underserviced areas may be enhanced, which could reduce morbidity, mortality, wait times and costs of health care delivery.

See the following video online:

Appendix 1: An obstetrician in Nova Scotia telementoring an individual in Bolivia on capturing real-time fetal ultrasound images. www.cmaj.ca/lookup/suppl/doi:10.1503/cmaj.120223/-/DC1 poor countries, where the greatest unmet needs in health care exist. ${ }^{2}$

Cellular networks have grown exponentially throughout the world. The latest survey by the International Telecommunication Union indicates that by $2011,90 \%$ of the world's population lived in areas serviced by cellular signals, and the number of cellphone subscriptions was approaching 6 billion, with the developing world accounting for two-thirds of this growth. ${ }^{3}$ Mobile broadband continues to increase, with $4 \mathrm{G}$ (fourth generation) connectivity rapidly becoming the norm, which allows for the transmission of more complex data. It is estimated that within the next 5 years, mobile Internet usage will surpass desktop-based usage. ${ }^{3}$ Mobile telemedicine technologies provide a portable platform capable of reaching any geographical location that has coverage by a cellphone signal.

Telemedicine applications using smartphones, tablets and other consumer mobile devices are increasingly being used for the transmission of medical data such as laboratory reports and diagnostic imaging. ${ }^{4} \mathrm{~A}$ recent systematic review of health care applications for smartphones documented 83 applications ranging from those focusing on disease diagnosis, drug reference and medical calculators to those focusing on remote monitoring of patients with chronic diseases such as diabetes and asthma. ${ }^{5}$ Smartphone applications for teleconsultation and postoperative follow-up are being explored and developed. However, smartphones have limitations because of restricted processing and memory capacity, as well as issues with communication security and privacy.

It is likely that dedicated devices (i.e., devices designed and used solely for telemedicine) will be required if remote-presence medical technology is to be relied on to inform decision-making that results in immediate clinical action by health professionals in clinical situations. The US Food and Drug Administration (FDA) has issued a rule regarding medical device data systems that clearly differentiates the handling of devices for 
medical display or documentation purposes from active patient monitoring. ${ }^{6}$ The next step in the evolution of mobile telemedicine is the development of portable, dedicated medical communication devices capable of providing real-time remote presence and transmission of diagnosticquality medical data from a range of peripheral diagnostic devices that will allow point-of-care therapeutic intervention. These devices must incorporate security, reliability and stable connectivity, and be able to pass stringent regulations.

\section{How do mobile remote-presence devices work?}

In contrast to smartphone applications, dedicated remote-presence medical devices have encryption protocols for secure communication, as well as high-resolution cameras and ports for the attachment of peripheral diagnostic devices such as portable ultrasound machines or digital stethoscopes (Figure 1). Although production of these devices is in its early stages, and each type of medical mobile device may be slightly different, the device that we are familiar with is the RPXpress (InTouch Health). This device is currently the only mobile remote-presence device designated by the FDA as a class II device, meaning it fulfills the FDA's requirements for active patient monitoring in clinical situations in which immediate clinical action may be required. The device is encased in a rugged protective shell that has a solid handle for easy manoeuvrability (Figure 2). It is the size of a regular laptop, weighs $7 \mathrm{lb}$ and is equipped with 2 screens and 2 cameras. Connectivity is provided by a standard wireless network and $3 \mathrm{G}$ cellphone signals with a minimum bandwidth of $300 \mathrm{Kbps}$ (kilobits per second). Box 1 summarizes our experience with this mobile remotepresence device.

\section{Who may benefit from mobile remote-presence devices?}

Remote-presence devices have the potential to be used in a wide range of applications for point-ofcare health care delivery, particularly in situations where medical expertise for the diagnosis and treatment of patients is required in real time. In addition to applications in the developing world, these devices have potential utility in emergency, mental health, and both highly specialized and primary care.

Mobile remote-presence devices have been used in highly specialized care, such as in long- distance programing of neuromodulation pulse generators implanted in patients with movement disorders and chronic pain. ${ }^{7}$ This type of application may have important implications in providing these patients with timely access to clinical expertise from the comfort of their home.

The use of remote-presence devices in emergency situations such as trauma assessment at the scene of an accident or during transport to a hospital setting could be an early application of this technology. For example, emergency physicians or trauma surgeons could perform realtime FAST (focused assessment with sonography for trauma) of the abdomen, thorax and heart in critically injured patients, which may result in reduced morbidity and mortality. ${ }^{8}$

Whereas the use of mobile remote-presence devices in emergency situations and highly specialized care makes intuitive sense, we think that a major impact of this technology could be in primary care applications, monitoring of patients with chronic disease and remote-presence house calls, in which a nurse or a paramedic uses the device to connect with the attending physician, who performs a teleconsultation with the use of peripheral attachments such as a digital stetho-

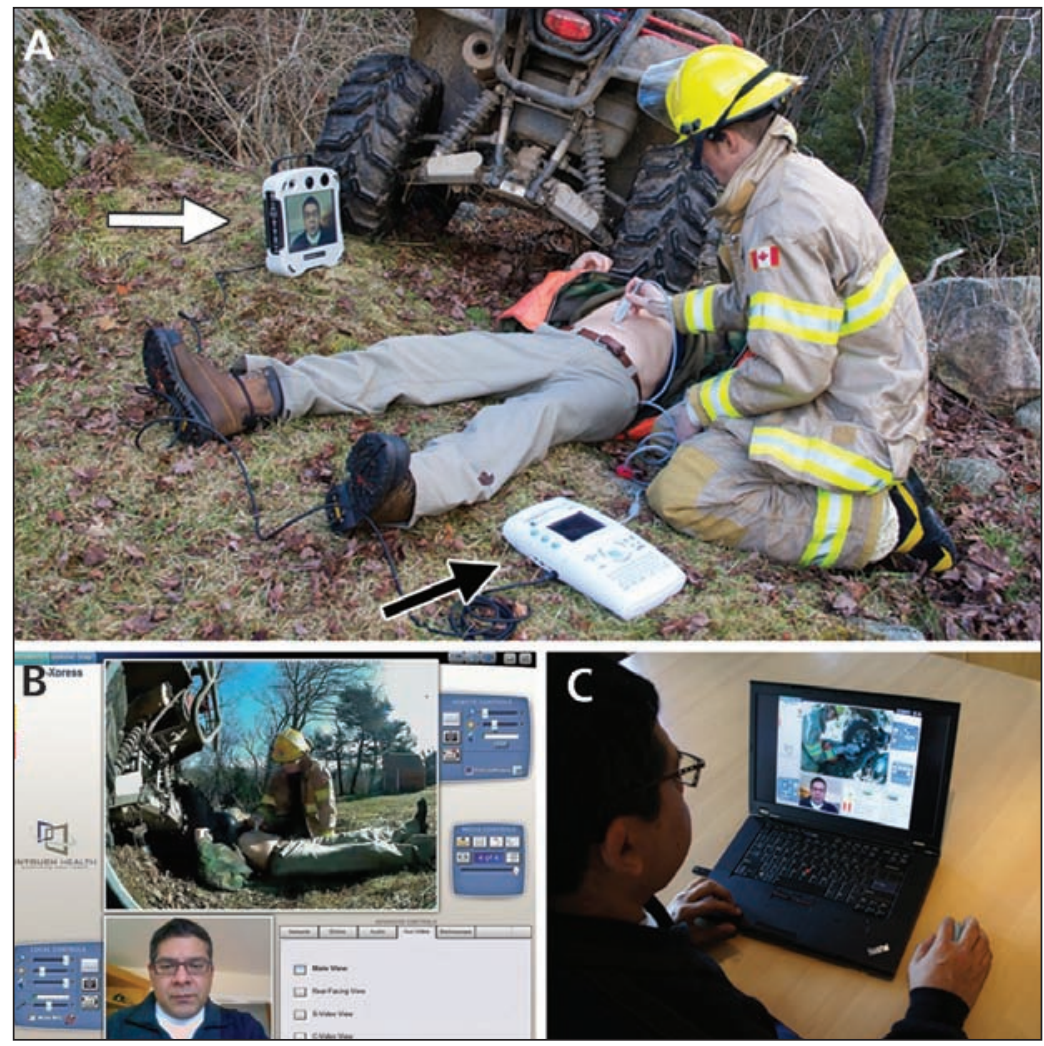

Figure 1: (A) Scene of a simulated accident showing the RP-Xpress (white arrow) and a portable ultrasound machine (black arrow). (B) Image of the screen of the RP-Xpress. This screen has 2 main displays that show the operating physician at the (C) control station, as well as the image of the remote location, allowing for early assessment and diagnosis of an injured person before arrival at the emergency department. 
scope, electrocardiography or ultrasonography. For example, mobile phones with built-in accelerometer sensors for home-based cardiac rehabilitation have been studied. ${ }^{9}$

Applications for mental health care in situations where there is limited or no access to psychiatrists may allow consulting psychiatrists to

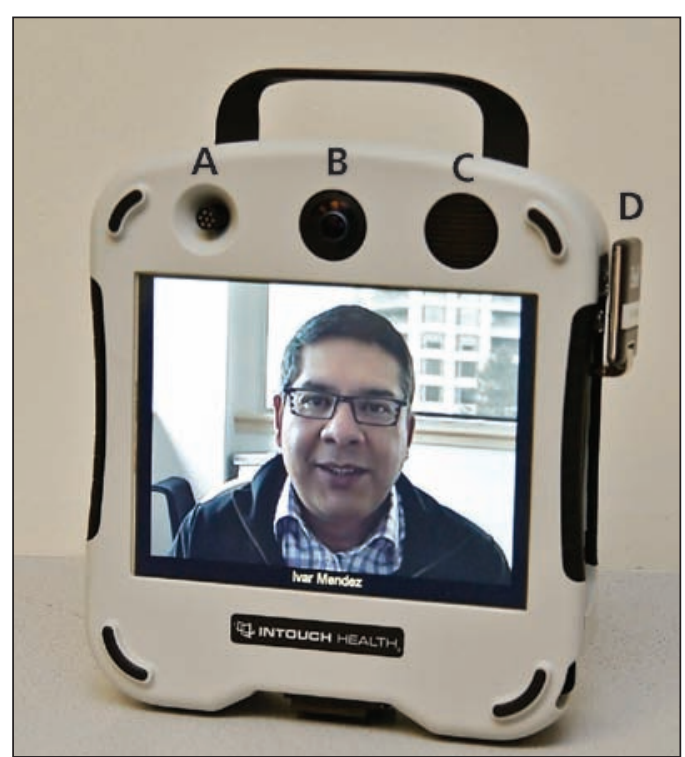

Figure 2: The (A) microphone, (B) high-resolution pan-tilt-zoom camera, (C) high-performance speakers and (D) cellular network modem of the RP-Xpress.

\section{Box 1: Our experience with a mobile remote-presence device}

We tested RP-Xpress in 2 settings: point-of-care trauma assessment in a simulated emergency situation and in prenatal assessments of women in remote, resource-poor areas of the Bolivian Andes.

We used the device in an outdoor, simulated accident scene (Figure 1) and during transport of the accident victim by ambulance to a referral centre. The aim of the test was to assess $3 \mathrm{G}$ cellphone connectivity, remote mentoring and real-time transmission of ultrasound and digital stethoscope data. The remote-presence scenario was hundreds of kilometres from the base control station, and the individuals assessing the subject had no experience in the operation of the portable ultrasound system. Connectivity was well maintained during the test, and we were able to perform FAST (focused assessment with sonography for trauma), as well as carotid ultrasonography.

We conducted pilot tests of prenatal assessment in the Bolivian Andes. The mobile-presence device was used to perform prenatal ultrasonography, assess the fetal heartbeat and provide expert opinion, in real time, from obstetricians located in Halifax, Nova Scotia, and La Paz, Bolivia. Bolivian cellphone networks were used for all of the trials.

An obstetrician in Halifax was able to connect to the portable device in Bolivia using his laptop control station. He was able to communicate, in real time, with a pregnant woman in Bolivia, hear the baby's heartbeat and, with the help of a nurse, perform a complete prenatal ultrasonographic assessment (Appendix 1, video available at www.cmaj.ca/lookup/suppl/doi :10.1503/cmaj.120223/-/DC1). The assessment lasted about 35 minutes, without any interruption in communication.

Several prenatal assessments of women were conducted in small health centres in rural areas by Bolivian obstetricians based in La Paz. The individuals operating the portable device in the remote locations were nurses or nurse's aides. In several remote locations, no electricity was available, and the systems operated exclusively on battery power. assess and follow up with patients in remote clinical settings or at home. The use of text messaging for the management of mental health disorders is currently being considered. ${ }^{10}$

Application of this technology in the developing world has considerable potential in promoting access to health care, collaboration and sharing of resources between different jurisdictions as well as knowledge transfer. We are currently exploring the value of this technology in reducing maternal mortality from preventable causes in the developing world (Box 1).

\section{What is the evidence so far?}

The most common and successful applications of telemedicine to date are teleradiology, telepathology and teledermatology. ${ }^{1}$ Some highincome countries have well-established services in these applications, whereas countries with smaller economies may have less developed applications of telemedicine. Mobile telemedicine programs such as Norway's teleECG initiative for real-time transmission of electrocardiograms to cardiologists have shown improved outcomes among patients with myocardial infarction. ${ }^{1}$

Fifty-seven health care applications for smartphones have been classified and analyzed critically in terms of their functionality. ${ }^{5}$ Although most direct clinical applications are in their early phases, the use of mobile phones to promote adherence to physical exercise guidelines has been shown to improve cardiac rehabilitation among outpatients. ${ }^{9}$ Furthermore, local wireless networks intended to coordinate relief efforts in disaster situations to optimize point-of-care diagnoses have been used in natural disaster settings, such as the 2010 earthquake in Haiti. ${ }^{11}$ The deployment of robust wireless networks was shown to enhance the efficiency of the disaster response by improving medical documentation, logistics, response coordination and communications. ${ }^{11}$

A number of pilot studies on mobile telemedicine technologies are being conducted, including one evaluating an application for diabetes monitoring used with a Global Positioning System (GPS) locator to alert health care providers to severe hypoglycemia in patients who require tight glycemic control and to transmit their location. ${ }^{12}$ Consumer cellphones and tablets are increasingly being used for transmitting medical information, and standard video conferencing and file-transfer services that are free to use, such as Skype, are being explored for real-time transmission of surgical wound images and diagnostic imaging, such as lung ultrasound. ${ }^{4,13}$

Innovative cellphone-based clinical microscopy capable of transmitting bright-field and 
fluorescent imaging has great potential in the diagnosis and screening of hematologic and infectious diseases in the developing world. It has been shown that this mobile technology can capture and transmit digital colour images of malaria parasites and sickled red blood cells in blood smears, as well as identify Mycobacterium tuberculosis in sputum smears. ${ }^{14}$

\section{What are the barriers to implementation?}

The most prevalent barrier to implementation of mobile remote-presence devices is likely to be the perception of high costs for the device and the connectivity. In our experience, the cost of the device itself was US\$25 000, and connectivity charges varied according to the jurisdiction in which the device was tested. We used a regular 3G USB modem provided by the local cellphone company in Canada and Bolivia. For our tests in Nova Scotia, we used a $\$ 60 /$ mo cellphone plan, and for about 14 hours of cellphone time in Bolivia, the cost was US\$18.

Obstacles for the provision of reliable remotepresence medicine include lack of stability of the cellular network signal and lack of broadband capacity in remote locations and underserviced countries. However, the increasing availability of cellular networks and the continuous increase in broadband capacity are likely to make this barrier less of an issue in the near future.

Expertise in computers and information technology in remote locations is less of a factor for use of mobile remote-presence devices than for telemedicine, which relies on computers and landbased Internet connectivity. The lack of medical expertise in a remote location to perform point-ofcare diagnosis is a relative barrier that could be mitigated by real-time telementoring. There is evidence that nonexperts can perform long-distance programming of neuromodulation devices or sophisticated diagnostic tests such as FAST with remote mentoring by experts. ${ }^{7.15}$

For health applications run by smartphones, another important issue is the need for standardization, because most applications are stand-alone applications and do not meet the requirements to be incorporated into hospital information systems or electronic medical records. ${ }^{5}$

Perhaps the most substantial barriers for mobile telemedicine are related to medical liability, jurisdictional legal considerations, provider remuneration, data and patient confidentiality, competing health priorities, and the lack of regional and national strategies and standards for implementation. A recent study that examined barriers to the implementation of robotic telemedicine has determined that the top barriers to adoption of telemedicine solutions in emergency and critical care are regulatory barriers for physician's privileges, financial barriers for billing of remote-presence services and resistance to changing established clinical paradigms. ${ }^{16}$

However, the explosive increase in the use of consumer mobile devices for medical applications may force streamlining of the regulatory and remuneration issues. Public expectations and pressure for cost-effective and decentralized provision of health care may play a substantial role in removing cultural barriers to remote-presence medicine, especially in underserviced communities. The acceptance by patients and their families of remote-presence solutions for delivery of health care is quite favourable. ${ }^{17}$

\section{What can we expect in the future?}

Mobile remote-presence devices for telemedicine have the potential to change the way health care is delivered in developed and developing nations. The availability of cellular network signals around the globe and rapidly increasing bandwidth will provide the telecommunication platform for a wide range of mobile telemedicine applications. The use of low-cost, dedicated remote-presence devices will increase access to medical expertise for anybody living in a geographical area with a cellphone signal. This access will be especially beneficial to people in rural or remote communities, such as northern regions of Canada, or in developing countries where medical expertise is insufficient or not available.

As technology continues to advance at a rapid pace, we can expect an increase in the sophistication and capabilities of mobile remotepresence devices and diagnostic peripheral attachments. Real-time blood chemistry analysis, portable imaging systems, electrophysiologic assessment tools and other diagnostic implements will be developed, which will increase our ability to perform point-of-care diagnosis. This switch from the current model of centralized diagnosis in large medical facilities to point-ofcare diagnosis could dramatically increase medical efficacy by removing barriers of time and distance, reducing wait times and decreasing the cost of health care delivery.

Mobile technologies could have a large impact on the delivery of health-behaviour interventions, such as encouraging smoking cessation, weight loss and physical activity. ${ }^{18}$ The development of sophisticated sensors that use wireless technology to communicate with mobile devices to track patients with chronic diseases or 
monitor an expanding number of vital parameters (e.g., oxygen saturation, cardiac rhythm, peak flow in asthma patients and blood glucose levels) may have a substantial effect on the forces that will drive health care in the future: major aging of the population, massive growth in chronic diseases and not enough caregivers. ${ }^{19}$

Although mobile telemedicine may be applied initially to emergency situations, remote locations and the developing world, its major impact may be in the delivery of primary health care. We can envision the use of mobile remotepresence devices by allied health personnel in a wide range of scenarios, from home care visits to follow-up sessions for mental health care, in which access to medical expertise in real time would be just a phone call away.

\section{References}

1. Telemedicine: opportunities and developments in member states: report on the Second Global Survey on eHealth, 2009. Geneva (Switzerland): World Health Organization; 2010.

2. Martínez A, Villarroel V, Seoane J, et al. Analysis of information and communication needs in rural primary health care in developing countries. IEEE Trans Inf Technol Biomed 2005;9:66-72.

3. The world in 2011 - ICT facts and figures. Geneva (Switzerland): International Telecommunication Union; 2011. Available: www.itu .int/en/ITU-D/Statistics/Documents/facts/ICTFactsFigures2011.pdf (accessed 2013 May 25).

4. Boulos MN, Wheeler S, Tavares C, et al. How smartphones are changing the face of mobile and participatory healthcare: an overview, with example from eCAALYX. Biomed Eng Online 2011;10:24

5. Mosa AS, Yoo I, Sheets L. A systematic review of healthcare applications for smartphones. BMC Med Inform Decis Mak 2012; 12:67.

6. United States Government Printing Office. Federal register: rules and regulations. Available: www.gpo.gov/fdsys/pkg/FR-2011-02 -15/pdf/2011-3321.pdf (accessed 2012 Nov. 19).

7. Mendez I, Song M, Chiasson P, et al. Point-of-care programming for neuromodulation: a feasibility study using remote presence. Neurosurgery 2013;72:99-108.
8. Moore CL, Copel JA. Point-of-care ultrasonography. $N$ Engl J Med 2011;364:749-57.

9. Walters DL, Sarela A, Fairfull A, et al. A mobile phone-based care model for outpatient cardiac rehabilitation: the care assessment platform (CAP). BMC Cardiovasc Disord 2010;10:5.

10. van den Berg N, Grabe HJ, Freyberger HJ, et al. A telephoneand text-message based telemedical care concept for patients with mental health disorders - study protocol for a randomized, controlled study design. BMC Psychiatry 2011;11:30.

11. Yu JN, Brock TK, Mecozzi DM, et al. Future connectivity for disaster and emergency point of care. Point Care 2010;9:185-92.

12. Dassau E, Jovanovic L, Doyle FJ III, et al. Enhanced 911/global position system wizard: a telemedicine application for the prevention of severe hypoglycemia - monitor, alert, and locate. J Diabetes Sci Technol 2009;3:1501-6.

13. McBeth PB, Crawford I, Blaivas M, et al. Simple, almost anywhere, with almost anyone: remote low-cost telementored resuscitative lung ultrasound. J Trauma 2011;71:1528-35.

14. Breslauer DN, Maamari RN, Switz NA, et al. Mobile phone based clinical microscopy for global health applications. PLOS ONE 2009; 4:e6320.

15. Boniface KS, Shokoohi H, Smith ER, et al. Tele-ultrasound and paramedics: real-time remote physician guidance of the Focused Assessment With Sonography for Trauma examination. Am J Emerg Med 2011;29:477-81.

16. Rogove HJ, McArthur D, Demaerschalk BM, et al. Barriers to telemedicine: survey of current users in acute care units. Telemed J E Health 2012;18:48-53.

17. Sucher JF, Todd SR, Jones SL, et al. Robotic telepresence: a helpful adjunct that is viewed favorably by critically ill surgical patients. Am J Surg 2011;202:843-7.

18. Riley WT, Rivera DE, Atienza AA, et al. Health behavior models in the age of mobile interventions: Are our theories up to the task? Transl Behav Med 2011;1:53-71.

19. McKenna M. The new age of medical monitoring. Sci Am 2013; 308:33-4.

Affiliations: Departments of Surgery, Division of Neurosurgery (Mendez); and Obstetrics and Gynaecology, Division of Maternal Fetal Medicine (Van den Hof), Dalhousie University, Halifax, NS

Contributors: Both authors wrote and revised the article, and approved the final version submitted for publication.

Acknowledgements: The authors thank Luis Bustamante for technical support, Tatjana Vukoja for preparing some of the figures, Roy Dempsey for photography and K. Adam Baker (Atlantic Clinical Consultants) for assistance with manuscript preparation. 